\title{
PREFACE: SPECIAL ISSUE FOR ITP 60TH ANNIVERSARY
}

The present issue is dedicated to the celebration of the 60th anniversary of the Kutateladze Institute of Thermophysics (ITP), Novosibirsk, Russia. This is a well-known research center where thermophysical phenomena have been investigated successfully by means of both theory and experiment. The expertise of the members of the Institute is clearly demonstrated by the high-quality papers included in the present issue.

In general, the research at ITP is devoted to heat and mass transfer in single- and multiphase systems. Fundamental problems of fluid mechanics include the deep understanding of vortex motion and wave dynamics. The classical book on wave flows of liquid films, by S.V. Alekseenko, V.E. Nakoryakov, and B.G. Pokusaev, introduced fluid mechanics and heat transfer in liquid films to a whole generation of researchers both in Russia and abroad. Professor V.E. Nakoryakov also initiated the research on heat and mass transfer in porous media, fluid mechanics of two-phase flows, and absorption thermal transformers. At the same time the research is performed on fundamental engineering phenomena such as those in heat power engineering. Boiling studies have been traditional areas of strength of ITP, starting with the pioneering works of S.S. Kutateladze on the hydrodynamic theory of boiling crisis, and continuing in several laboratories to the present day. The problem of turbulence is investigated in the frameworks of fundamental physics and applied engineering, among many other topics. S.S. Kutateladze and A.I. Leontiev developed a theory of turbulent boundary layers with vanishing viscosity, leading to efficient computational methods for engineering applications. Studies of alloys of alkali metals used in nuclear reactors were conducted starting from the early days of the Institute of Thermophysics under the direction of I.I. Novikov, a founder of this new research direction in applied technical thermodynamics. Vacuum chambers developed by A.K. Rebrov and his group have been used for a wide range of studies of rarefied gases with important aerospace applications. Fundamental studies of plasma generation conducted at the Institute resulted in development of high-efficiency plasmatrons.

There are several well-known Russian journals on heat and mass transfer and researchers at the ITP have a consistent record of publications in them. However, they are also very active publishing in journals of Europe and North America. In this way, their theoretical and experimental results have been widely available to the community and we are all aware of the high standards of their papers. Foreign researchers are also able to meet them at a number of conferences and symposia around the world and exchange mutual impressions of their results. Several members of the ITP, along with other renowned scientists from different countries, began the organization of the International Topical Team Workshop (ITTW) to gather experts from all over the world with an interest on theoretical and experimental fluid mechanics related to processes in the presence of gravity, in the absence of gravity, or under microgravity conditions. The first ITTW was held in Brussels, Belgium, in 2006. This year, the 13th conference in the series was held in Xi' an, China. Several lectures were related to the preparation of experiments to be conducted at the International Space Station, in parabolic flights, or in a drop tower.

The participants of ITTW were invited to submit their work to the journal Microgravity Science and Technology. In a few years, this became a tradition, but the organizers recognized that a good number of papers were not related to microgravity conditions. In fact, a number of presentations were related to phenomena under terrestrial conditions. Besides, problems where an interface played an important role were the subject of many of the lectures given in ITTW. Therefore, the idea of Professors Sergey Aleeksenko and Oleg Kabov of the ITP, together with other researchers from different countries, was to launch a new journal which may include research on interfacial phenomena under terrestrial conditions, but not excluding problems under other gravitational conditions. In this way, they promoted the concept of this new journal with their colleagues around the world in 2012 and received wide support. Professor Kabov then proposed these ideas to the president of Begell House, Yelena Shafeyeva. Begell House is a successful publisher mainly focused on high-quality academic work. The result of the proposal was that the new journal Interfacial Phenomena and Heat Transfer was launched in the beginning of 2013. The Editor-in-Chief is Professor Oleg Kabov from the ITP and the team of Associate Editors includes representatives from Canada, China, France, Japan, Mexico, and the United 
States. The Editorial Board includes well-known scientists from many countries of the world showing the cultural and scientific diversity of the journal. The Institute of Thermophysics is represented by the Editor-in-Chief, but also by Professors S.V. Alekseenko and D.M. Markovich, the Director of the Institute of Thermophysics.

Therefore, we are gathered here, taking part in the journal because we believe in the solid scientific background and tradition of the Kutateladze Institute of Thermophysics. We are very happy to celebrate its 60th anniversary and we wish many happy returns to every one of its members.

Guest Editors:

Vladimir S. Ajaev

Southern Methodist University,

Dallas, Texas, USA

Hang Guo

MOE Key Laboratory of Enhanced Heat

Transfer and Energy Conservation; Beijing Key Laboratory of Heat Transfer and Energy Conversion, College of Environmental and Energy Engineering, Beijing University of Technology, Beijing 100124, China
Luis Antonio Dávalos-Orozco

Instituto de Investigaciones en Materiales, Departamento de Polímeros, Universidad Nacional Autónoma de México, Ciudad Universitaria, Circuito Exterior S/N

Delegación Coyoacán, 10000 México D.F., México

Haruhiko Ohta

Department of Aeronautics and Astronautics, Faculty of Engineering, Kyushu University

Motooka 744, Nishi-ku, Fukuoka 819-0395, Japan 